\title{
Comparison of CFD and DSMC Using Calibrated Transport Parameters
}

\author{
D.S. Liechty ${ }^{1, \text { a) }}$, A.J. Wise ${ }^{1, \text { b) }}$, S. Subramaniam ${ }^{2, c)}$ and K.A. Stephani ${ }^{2, \text { d) }}$ \\ ${ }^{1}$ Aerothermodynamics Branch, NASA Langley Research Center, 16 Victory Street Hampton, VA 23681, USA \\ ${ }^{2}$ Department of Mechanical Science and Engineering, University of Illinois at Urbana-Champaign, Urbana, IL, \\ 61801, USA \\ a)Derek.S.Liechty@nasa.gov \\ b)A.J.Wise@nasa.gov \\ c) $\underline{\text { ksteph@illinois.edu }}$ \\ d) $\underline{\text { ssbrmnm3@illinois.edu }}$
}

\begin{abstract}
Hypersonic re-entry flows span a wide range of length scales where regions of both rarefied and continuum flow exist. Traditional computational fluid dynamics (CFD) techniques do not provide an accurate solution for the rarefied regions of such 'mixed' flow fields. Although direct simulation Monte Carlo (DSMC) can be used to accurately capture both the continuum and rarefied features of 'mixed' flow fields, they are computationally expensive when employed to simulate the low Knudsen number continuum regimes. Thus, a hybrid framework for seamlessly combining the two methodologies, CFD and DSMC, continues to be a topic of significant research effort. Ensuring consistency in the reaction kinetics and transport models employed within CFD and DSMC is a crucial requirement for obtaining a reliable solution from a hybrid framework for combined continuum/rarefied high speed flows. This paper represents one of the first studies to utilize the calibrated transport parameters developed to ensure consistency between CFD and DSMC solvers. The new variable soft sphere (VSS) parameters are compared to both previous "standard" variable hard sphere (VHS) parameters and also to solutions from the CFD transport properties that the new parameters were developed to reproduce.
\end{abstract}

\section{INTRODUCTION}

Computational techniques allow for the prediction of forces, moments, and heating experienced by a re-entry vehicle all along its trajectory, during the design stage of the vehicle. For an accurate, yet computationally efficient estimate of flow field quantities, the simulation methodology employed must vary along the trajectory depending on the degree of rarefaction within the flow field. Computational fluid dynamics (CFD) solutions based on the NavierStokes (NS) equations provide an accurate description of continuum effects, but a particle-based solution methodology, such as direct simulation Monte Carlo (DSMC), is required to capture the non-equilibrium phenomena in regions where the NS assumptions break down, such as within the shock, boundary layer and wake region of the vehicle. While DSMC is capable of simulating the continuum regions as well, this can become prohibitively expensive computationally. For these reasons, there have been many attempts over the years to perform hybrid CFD/DSMC simulations to provide a single flow field solution ${ }^{1-4}$.

Efforts have recently begun to develop a hybrid framework for combined continuum/rarefied high speed flows ${ }^{4-6}$ that is coupled in physical space. The first step is to be able to accurately define locations in the flow field where continuum breakdown is occurring, by employing continuum breakdown parameters. These locations serve as 'boundaries' to transition between the CFD and DSMC methodology. Continuum breakdown parameters that capture the contribution of multiple physical mechanisms that can lead to breakdown have been formulated for chemically reacting flows ${ }^{6}$. Thus, based on the value of these breakdown parameters with respect to a breakdown threshold, boundaries for CFD/DSMC transition are identified. Once a boundary has been defined, an approach for the 
generation of particles has been developed in which the fluxes of mass, momentum, and energy determined from the NS solution are used to prescribe the appropriate velocity distribution function to be used within the DSMC particle generation process ${ }^{4}$. The next challenge is to ensure consistency between CFD and DSMC with respect to transport properties and other non-equilibrium phenomena, such as internal energy relaxation and chemical processes $\mathrm{A}$ systematic approach for calibrating the DSMC collision model parameters to achieve consistency in the transport process was therefore developed and has been used to generate best-fit variable hard sphere (VHS) and variable soft sphere (VSS) parameter values for air systems 5 .

The current paper focuses on the implementation of the calibrated collision model parameters. Specifically, the chemically-frozen flow of an oxygen (2-species) and an air system (5- and 11-species) around a cylinder, as described in Ref. 7, at Mach numbers of 10 and 25 are investigated, and flow field and surface parameters are compared between the DSMC and CFD solutions. The MAP DSMC code ${ }^{8}$ was used for all DSMC simulations. In addition to the recommended best-fit VSS parameter values from Ref. 5, multiple additions have been previously implemented to more closely match CFD results for this and future comparisons, including the addition of electronic energy levels, improvements in the implementation of the Millikan-White vibrational relaxation model, and the inclusion of a statistical mechanics-based method of computing reverse reaction rates. Various parameters are varied in the DSMC simulations from previous best practices to what are considered NASA's new best practices. The CFD solutions were generated using the LAURA code ${ }^{9}$.

\section{TRANSPORT PROPERTIES AND MODEL PARAMETERS}

It is important to ensure consistency between computational transport models used in flow solvers when making comparisons of flow field solutions, especially when considering a hybrid CFD/DSMC framework. The treatment of the transport properties in CFD and DSMC solvers is decidedly different. The transport properties in CFD solvers are obtained from expressions involving the collision integrals that are stored as curve fits as a function of temperature ${ }^{10}$, and the transport properties are calculated from the collision integrals using either the full Chapman Enskog formulation or through mixing rules ${ }^{11}$. The transport properties in DSMC simulations, however, are obtained as a result of the transport of mass, momentum, and energy during the collision process between the simulated particles. The details of the collision process are dictated by the phenomenological collision cross section models used in DSMC. These cross sections can then be used to compute the collision integrals expressed in terms of the DSMC collision model parameters, thereby providing a consistent means for quantification and comparison of the transport coefficients in CFD and DSMC. The transport coefficients can then be computed in a similar manner for both CFD and DSMC using the Chapman-Enskog theory.

There are several collision models available in DSMC, but the most commonly used models are the variable hard sphere (VHS) ${ }^{12}$ and the variable soft sphere (VSS) ${ }^{13}$ models, and these are the models under consideration. In both of these models, the particles are approximated as a hard sphere of diameter $d$ which is a function of the relative speed of the collision $g$,

$$
d=d_{\text {ref }}\left(\frac{g_{\text {ref }}}{g}\right)^{(\omega-1 / 2)}
$$

The variables $d_{r e f}$ and $g_{r e f}$ are the reference values defined at a particular reference temperature $T_{r e f}$ and $\omega$ is the temperature exponent controlling the particle's diameter with temperature. In the VHS model, the scattering is assumed to be isotropic, but in the VSS model the scattering angle $\chi$ can be varied,

$$
\chi=2 \cos ^{-1}\left\{(b / d)^{1 / \alpha}\right\}
$$

where $b$ is the impact parameter and $\alpha$ is referred to as the scattering exponent that introduces anisotropic scattering in the center-of-mass frame of reference. This parameter allows the VSS model to provide consistent results for both diffusion and viscous cross section in comparison with the inverse power law potential.

The collision integrals and the ratio of collision integrals can be expressed ${ }^{5}$ in terms of the VHS parameters as:

$$
\left.\Omega_{s t}^{1}(1)\right|_{V H S}=\frac{\pi}{2} d_{r e f}^{2}\left(\frac{k_{b} T}{2 \pi m_{s t}^{*}}\right)^{1 / 2}\left(\frac{T_{r e f}}{T}\right)^{\omega-1 / 2}\left[\frac{\Gamma(7 / 2-\omega)}{\Gamma(5 / 2-\omega)}\right]
$$




$$
\begin{gathered}
\left.\Omega_{s t}^{2}(2)\right|_{V H S}=\frac{\pi}{3} d_{r e f}^{2}\left(\frac{k_{b} T}{2 \pi m_{s t}^{*}}\right)^{1 / 2}\left(\frac{T_{r e f}}{T}\right)^{\omega-1 / 2}\left[\frac{\Gamma(9 / 2-\omega)}{\Gamma(5 / 2-\omega)}\right] \\
\left.B_{s t}\right|_{V H S}=\frac{5 \Gamma(9 / 2-\omega)-\Gamma(11 / 2-\omega)}{5 \Gamma(7 / 2-\omega)}
\end{gathered}
$$

where $k_{b}$ is the Boltzmann constant, $T$ is the temperature, $m_{s t}{ }^{*}$ is the reduced mass, and $\Gamma(x)$ is the gamma function. The collision integrals are then expressed in terms of the VSS parameters as:

$$
\begin{gathered}
\left.\Omega_{s t}^{1}(1)\right|_{V S S}=\left.\left(\frac{2}{\alpha+1}\right) \Omega_{s t}^{1}(1)\right|_{V H S} \\
\left.\Omega_{s t}^{2}(2)\right|_{V S S}=\left.\left[\frac{6 \alpha}{(\alpha+1)(\alpha+2)}\right] \Omega_{s t}^{2}(2)\right|_{V H S}
\end{gathered}
$$

The ratio of collision integrals $\left.B_{s t}\right|_{V S S}$ is the same as that for $\left.B_{S t}\right|_{V H S}$ given in Eq. 5.

The mixture viscosity from the VHS and VSS models were determined ${ }^{14}$ from the first-order Chapman-Enskog approximation of the mixture viscosity, defined as:

$$
[\mu]_{1}=\sum_{s} b_{s}
$$

where $b_{s}$ is the contribution of each species to the overall mixture viscosity and may be determined by solving the following system:

$$
\chi_{s}=b_{s}\left\{\frac{\chi_{s}}{\left[\mu_{s}\right]_{1}}+\sum_{t \neq s} \frac{3 \chi_{t}}{\left(\rho_{s}^{\prime}+\rho_{t}^{\prime}\right) \mathcal{D}_{s t}}\left(\frac{2}{3}+\frac{m_{t}}{m_{s}} A_{s t}\right)\right\}-\chi_{s} \sum_{t \neq s} \frac{3 b_{t}}{\left(\rho_{s}^{\prime}+\rho_{t}^{\prime}\right) \mathcal{D}_{s t}}\left(\frac{2}{3}-A_{s t}\right)
$$

where $\chi$ is the mole fraction of the species, and $\left[\mu_{s}\right]_{1}, A_{s t}$, and $\mathcal{D}_{s t}$ are defined as:

$$
\begin{gathered}
{\left[\mu_{s}\right]_{1}=\frac{5 k_{b} T}{8 \Omega_{s s}^{2}(2)}} \\
A_{s t}=\frac{\Omega_{s t}^{2}(2)}{5 \Omega_{s t}^{1}(1)} \\
\mathcal{D}_{s t}=\frac{3 k_{b} T}{16 n m_{s t}^{*} \Omega_{s t}^{1}(1)}
\end{gathered}
$$

The terms $\rho_{s}^{\prime}$ and $\rho_{t}^{\prime}$ refer to the density of species $s$ and $t$ when pure at the pressure and temperature of the actual gas mixture, $m_{s}$ and $m_{t}$ are the masses of species $s$ and $t$, and $n$ is the number density.

The mixture translational thermal conductivity was shown to be determined by ${ }^{14}$ :

$$
\left[K_{t r}\right]_{1}=\sum_{s} a_{s}
$$

where $a_{s}$ is the contribution of each species to the overall mixture translational thermal conductivity and may be determined by solving the following system:

$$
\begin{aligned}
\chi_{s}=a_{s}\left\{\frac{\chi_{s}}{\left[\lambda_{s}\right]_{1}}+\right. & \left.\sum_{t \neq s} \frac{T \chi_{t}}{5 p \mathcal{D}_{s t}}\left(6\left[\frac{m_{s}}{m_{s}+m_{t}}\right]^{2}+\left(5-4 B_{s t}\right)\left[\frac{m_{t}}{m_{s}+m_{t}}\right]^{2}+8 \frac{m_{s} m_{t}}{\left(m_{s}+m_{t}\right)^{2}} A_{s t}\right)\right\} \\
& -\chi_{s} \sum_{t \neq s} a_{t}\left[\left(T \frac{m_{s} m_{t}}{\left(m_{s}+m_{t}\right)^{2}}\right) / 5 p \mathcal{D}_{s t}\right]\left(11-4 B_{s t}-8 A_{s t}\right)
\end{aligned}
$$

where $\left[\lambda_{s}\right]_{1}$ is defined as:

$$
\left[\lambda_{s}\right]_{1}=\frac{25 c_{v, s, t r} k_{b} T}{16 \Omega_{S S}^{2}(2)}
$$


where $c_{v}$ is the specific heat at constant volume of species $s$, and $p$ is the pressure of the gas mixture.

The rotational, vibrational, and electronic conductivities are determined using Eucken's formula, as done in Ref. 14. The internal thermal conductivities are given by

$$
\begin{aligned}
K_{\text {rot }} & =\sum_{i \in s} \frac{\rho_{i} c_{v, r o t, i}}{\sum_{j \in s} \chi_{j} / \mathcal{D}_{i j}} \\
K_{v i b} & =\sum_{i \in s} \frac{\rho_{i} c_{v, v i b, i}}{\sum_{j \in s} \chi_{j} / \mathcal{D}_{i j}} \\
K_{\text {ele }} & =\sum_{i \in s} \frac{\rho_{i} c_{v, \text { ele }, i}}{\sum_{j \in s} \chi_{j} / \mathcal{D}_{i j}}
\end{aligned}
$$

where $\rho_{i}$ is the partial density defined as the product of the mixture density, $\rho$, and the species mass fraction, $Y_{i}$

$$
\rho_{i}=\rho Y_{i}
$$

The specific heats are calculated from

$$
c_{v}=\frac{\zeta k}{2 m}
$$

where $\zeta$ is the number of degrees of freedom. The number of degrees of freedom are well defined for the translational, rotational, and vibrational components and are

$$
\begin{gathered}
\zeta_{\text {trans }}=3 \\
\zeta_{\text {rot }}=2 \\
\zeta_{v i b}=\frac{2 \Theta_{v i b} / T}{\exp \left(\Theta_{v i b} / T\right)-1}
\end{gathered}
$$

The number of electronic degrees of freedom are not quite so well defined. We know that ${ }^{18,19}$

$$
\begin{gathered}
e_{e l}=\frac{1}{2} \zeta_{e l} R T_{e l}=R T^{2} \frac{\partial}{\partial T} \ln Q_{e l} \\
Q_{e l}=\sum_{i} g_{i} e^{-\Theta_{i} / T}
\end{gathered}
$$

where $e_{e l}$ is the electronic energy, $R$ is the gas constant, $T_{e l}$ is the electronic temperature, $Q_{e l}$ is the electronic partition function, $g_{i}$ is the degeneracy of level $i, \Theta_{i}=\varepsilon_{i} / k$ is the characteristic temperature of energy level $i$, and $\varepsilon_{i}$ is the energy of energy level $i$. Solving for the electronic degrees of freedom, we get

$$
\zeta_{e l}=\frac{2 \sum_{i} g_{i} \Theta_{i} e^{-\Theta_{i} / T}}{T_{e l} \sum_{i} g_{i} e^{-\Theta_{i} / T}}
$$

where the sum should be taken over all electronic energy levels. However, the assumption is often made that only the first two energy levels are sufficiently active, so the sum is frequently only taken over the first two energy levels. This will be examined later in this work.

Previous work ${ }^{5}$ has provided a recommended set of DSMC collision model parameters for both neutral (6-species) and ionized (13-species) gas mixtures based on calibrations against $a b$ initio-based collision integral data. The VHS and VSS parameters are input quantities that may be specified for each species, or for each collision pair. If the parameters are specified for each species only, it is referred to as collision-averaged pairing and the parameters for the colliding pair are taken as the average values of the two species. When the parameters are specified for each specific collision pair, then it is referred to as collision-specific pairing. It was shown by Stephani et al. ${ }^{14}$ that the collision-specific pairing provides the most accurate and physically consistent description of the collision processes between unlike species. The recommended set of values for the VSS model parameters, given in Ref. 5, are presented in Tables 1-4 for 13-species ionized air. The suggested values given for 6-species neutral air are not considered here for consistency and the extended range of valid temperatures of the 13-species calibration. The parameters for 13species ionized air become more complex due to the charged particle interactions. The parameters for the neutralneutral interactions are listed in Table 1 and neutral-charged interactions in Table 2. Due to the variation of neutral- 
electron collision integrals with respect to temperature, separate temperature ranges must be defined and the VSS model parameters are listed for these collisions in Table 3. Finally, the VSS model parameters controlling the interactions between charged particles are given as curve fits as a function of electron number density in Table 4 . The reader is encouraged to refer to Ref. 5 for the VHS parameters and the 6-species neutral air parameters.

TABLE 1. Collision-specific VSS parameters for neutral-neutral interactions for 13-species ionized air.

\begin{tabular}{ccccccc}
\hline $\mathrm{d}_{\mathrm{ref}}(\AA)$ & $\mathrm{N}_{2}$ & $\mathrm{O}_{2}$ & $\mathrm{NO}$ & $\mathrm{N}$ & $\mathrm{O}$ & $\mathrm{Ar}$ \\
\hline $\mathrm{N}_{2}$ & 4.040 & 3.604 & 4.391 & 4.088 & 3.222 & 3.882 \\
$\mathrm{O}_{2}$ & & 3.896 & 4.054 & 3.721 & 3.734 & 3.972 \\
$\mathrm{NO}$ & & & 4.218 & 4.028 & 3.693 & 4.049 \\
$\mathrm{~N}$ & & & & 3.697 & 3.811 & 3.963 \\
$\mathrm{O}$ & & & & & 3.692 & 4.022 \\
$\mathrm{Ar}$ & & & & & & 4.011 \\
\hline \multicolumn{1}{c}{} & & & & & \\
\hline$\omega$ & $\mathrm{N}_{2}$ & $\mathrm{O}_{2}$ & $\mathrm{NO}$ & $\mathrm{N}$ & $\mathrm{O}$ & $\mathrm{Ar}$ \\
\hline $\mathrm{N}_{2}$ & 0.686 & 0.703 & 0.756 & 0.762 & 0.702 & 0.703 \\
$\mathrm{O}_{2}$ & & 0.700 & 0.718 & 0.757 & 0.760 & 0.719 \\
$\mathrm{NO}$ & & & 0.737 & 0.788 & 0.752 & 0.719 \\
$\mathrm{~N}$ & & & & 0.790 & 0.794 & 0.784 \\
$\mathrm{O}$ & & & & & 0.803 & 0.757 \\
$\mathrm{Ar}$ & & & & & & 0.722 \\
\hline & & & & & & \\
\hline$\alpha$ & $\mathrm{N}_{2}$ & $\mathrm{O}_{2}$ & $\mathrm{NO}$ & $\mathrm{N}$ & $\mathrm{O}$ & $\mathrm{Ar}$ \\
\hline $\mathrm{N}_{2}$ & 1.424 & 1.430 & 1.515 & 1.585 & 1.427 & 1.446 \\
$\mathrm{O}_{2}$ & & 1.463 & 1.501 & 1.567 & 1.542 & 1.467 \\
$\mathrm{NO}$ & & & 1.542 & 1.641 & 1.555 & 1.463 \\
$\mathrm{~N}$ & & & & 1.486 & 1.545 & 1.632 \\
$\mathrm{O}$ & & & & & 1.582 & 1.570 \\
$\mathrm{Ar}$ & & & & & & 1.470 \\
\hline & & & & &
\end{tabular}

TABLE 2. Collision-specific VSS parameters for neutral-charged interactions for 13-species ionized air.

\begin{tabular}{|c|c|c|c|c|c|c|}
\hline $\mathrm{d}_{\mathrm{ref}}(\AA)$ & $\mathrm{N}_{2}$ & $\mathrm{O}_{2}$ & $\mathrm{NO}$ & $\mathrm{N}$ & $\mathrm{O}$ & $\mathrm{Ar}$ \\
\hline $\mathrm{N}_{2}{ }^{+}$ & 4.975 & 4.034 & 4.100 & 3.987 & 3.823 & 5.384 \\
\hline $\mathrm{O}_{2}{ }^{+}$ & 3.434 & 5.245 & 3.417 & 3.155 & 2.990 & 4.678 \\
\hline $\mathrm{NO}^{+}$ & 3.752 & 3.714 & 5.325 & 3.584 & 3.382 & 5.002 \\
\hline $\mathrm{N}^{+}$ & 7.666 & 3.528 & 3.595 & 6.462 & 7.525 & 3.581 \\
\hline $\mathrm{O}^{+}$ & 3.477 & 3.453 & 3.469 & 7.092 & 5.625 & 3.461 \\
\hline $\mathrm{Ar}^{+}$ & 4.662 & 4.989 & 4.639 & 4.746 & 4.577 & 5.522 \\
\hline$\omega$ & $\mathrm{N}_{2}$ & $\mathrm{O}_{2}$ & $\mathrm{NO}$ & $\mathrm{N}$ & $\mathrm{O}$ & $\mathrm{Ar}$ \\
\hline $\mathrm{N}_{2}{ }^{+}$ & 0.601 & 0.660 & 0.658 & 0.657 & 0.660 & 0.777 \\
\hline $\mathrm{O}_{2}{ }^{+}$ & 0.699 & 0.670 & 0.695 & 0.682 & 0.682 & 0.834 \\
\hline $\mathrm{NO}^{+}$ & 0.676 & 0.677 & 0.653 & 0.668 & 0.666 & 0.800 \\
\hline $\mathrm{N}^{+}$ & 1.034 & 0.682 & 0.681 & 0.741 & 1.064 & 0.677 \\
\hline $\mathrm{O}^{+}$ & 0.691 & 0.695 & 0.688 & 0.995 & 0.725 & 0.685 \\
\hline $\mathrm{Ar}^{+}$ & 0.768 & 0.803 & 0.764 & 0.789 & 0.795 & 0.619 \\
\hline$\alpha$ & $\mathrm{N}_{2}$ & $\mathrm{O}_{2}$ & $\mathrm{NO}$ & $\mathrm{N}$ & $\mathrm{O}$ & $\mathrm{Ar}$ \\
\hline $\mathrm{N}_{2}{ }^{+}$ & 0.254 & 1.284 & 1.269 & 1.256 & 1.286 & 1.352 \\
\hline $\mathrm{O}_{2}^{+}$ & 1.378 & 0.157 & 1.365 & 1.339 & 1.366 & 1.490 \\
\hline $\mathrm{NO}^{+}$ & 1.343 & 1.343 & 0.209 & 1.313 & 1.336 & 1.426 \\
\hline $\mathrm{N}^{+}$ & 1.862 & 1.344 & 1.329 & 0.158 & 1.798 & 1.331 \\
\hline $\mathrm{O}^{+}$ & 1.365 & 1.359 & 1.352 & 1.585 & 0.164 & 1.353 \\
\hline $\mathrm{Ar}^{+}$ & 1.406 & 1.442 & 1.397 & 1.403 & 1.438 & 0.162 \\
\hline
\end{tabular}


TABLE 3. Collision-specific VSS parameters for neutral-electron interactions for 13-species ionized air.

\begin{tabular}{ccccc}
\hline & Temperature range $(\mathrm{K})$ & $\mathrm{d}_{\text {ref }}(\AA)$ & $\omega$ & $\alpha$ \\
\hline $\mathrm{N}_{2}$ & $5000-7400$ & 1.777 & 0.446 & 1.011 \\
& $7400-20000$ & 2.867 & 0.677 & 1.056 \\
$\mathrm{O}_{2}$ & $5000-10300$ & 1.360 & 0.455 & 1.142 \\
& $10300-20000$ & 1.632 & 0.534 & 1.285 \\
$\mathrm{NO}$ & $5000-20000$ & 3.790 & 0.834 & 1.250 \\
$\mathrm{~N}$ & $5000-20000$ & 3.863 & 0.842 & 1.983 \\
$\mathrm{O}$ & $5000-20000$ & 0.888 & 0.327 & 1.838 \\
$\mathrm{Ar}$ & $5000-20000$ & 0.193 & -0.434 & 1.801 \\
\hline
\end{tabular}

TABLE 4. Curve-fit coefficients for VSS collision-specific parameters as a function of electron number density $\left(n_{e}\right)$ for chargecharge interactions for 13 -species ionized air.

\begin{tabular}{|c|c|c|c|c|c|c|}
\hline \multirow[b]{2}{*}{ VSS parameter } & \multicolumn{3}{|c|}{ Attractive Potential } & \multicolumn{3}{|c|}{ Repulsive Potential } \\
\hline & $\mathrm{A}$ & $\mathrm{B}$ & $\mathrm{C}$ & $\mathrm{A}$ & $\mathrm{B}$ & $\mathrm{C}$ \\
\hline $\mathrm{d}_{\mathrm{ref}}(\AA)$ & $3.614 \times 10^{1}$ & $-3.294 \times 10^{3}$ & $7.497 \times 10^{4}$ & $4.794 \times 10^{1}$ & $-4.121 \times 10^{3}$ & $8.879 \times 10^{4}$ \\
\hline$\omega$ & $-3.636 \times 10^{-3}$ & $2.228 \times 10^{-1}$ & $-1.102 \times 10^{0}$ & $-4.298 \times 10^{-3}$ & $2.573 \times 10^{-1}$ & $-1.543 \times 10^{0}$ \\
\hline$\alpha$ & $3.846 \times 10^{-3}$ & $-1.573 \times 10^{0}$ & $6.279 \times 10^{1}$ & $3.489 \times 10^{-2}$ & $-3.659 \times 10^{0}$ & $9.661 \times 10^{1}$ \\
\hline
\end{tabular}

$\left(d_{\text {ref }}, \omega, \alpha\right)=A * \log ^{2}\left(n_{e}\right)+B * \log \left(n_{e}\right)+C$

\section{APPLICATION TO HYPERSONIC BLUNT BODY FLOW}

The two-dimensional, 12-inch diameter cylinder used by Lofthouse et $a{ }^{7}{ }^{7}$ is used as the geometry in the current study. The test case is a two-dimensional hypersonic flow of an oxygen system and an air system over a range of Mach numbers set such that the nominal free stream Mach numbers are 10 and 25 for a standard air composition (actual free stream Mach numbers will vary due to changes in gas composition). A nominal Knudsen number based on the cylinder diameter of 0.0005 was chosen to reduce any rarefaction effects, so a constant value of $n_{\infty}=1.00 \times 10^{22}$ $\mathrm{m}^{-3}$ was used for the free stream number density. The focus of the current study are the transport properties; therefore, the gas is assumed to be chemically frozen at post-shock equilibrium concentrations (as computed by the CEA code ${ }^{15}$ ) and thermal equilibrium is assumed for the CFD solutions and encouraged in the DSMC solutions by setting the internal energy collision numbers to a value of one (relaxation is performed for each particle at each collision). The free stream conditions are listed in Table 5. The species concentrations are listed in Tables 6 and 7 for the oxygen and air systems, respectively. The VSS results are obtained using the parameters listed in the above section. The VHS values are obtained using the standard high temperature air values distributed with the DAC software ${ }^{16,17}$. Flow field and surface distributions are discussed in the following sections.

TABLE 5. Free stream and surface conditions.

\begin{tabular}{ccccc}
\hline $\begin{array}{c}\text { Mach } \\
\text { Number }\end{array}$ & $u_{\infty}(\mathrm{m} / \mathrm{s})$ & $T_{\infty}(\mathrm{K})$ & $T_{\text {wall }}(\mathrm{K})$ & $\begin{array}{c}\text { Wall Boundary } \\
\text { Condition }\end{array}$ \\
\hline 10 & 2836 & 200 & 500 & Non-catalytic \\
25 & 7090 & 200 & 1500 & Non-catalytic \\
\hline
\end{tabular}

TABLE 6. Oxygen system compositions (mole fractions).

\begin{tabular}{ccc}
\hline & Mach 10 & Mach 25 \\
\hline $\mathrm{O}_{2}$ & $8.3736 \times 10^{-1}$ & 0.0 \\
$\mathrm{O}$ & $1.6264 \times 10^{-1}$ & 1.0 \\
\hline
\end{tabular}

TABLE 7. Air system compositions (mole fractions).

\begin{tabular}{ccc}
\hline & Mach 10 & Mach 25 \\
\hline $\mathrm{O}_{2}$ & $1.5617 \times 10^{-1}$ & $7.9000 \times 10^{-4}$ \\
$\mathrm{~N}_{2}$ & $7.8049 \times 10^{-1}$ & $2.2447 \times 10^{-1}$ \\
$\mathrm{O}$ & $1.1267 \times 10^{-1}$ & $2.8924 \times 10^{-1}$ \\
$\mathrm{~N}$ & $1.0000 \times 10^{-5}$ & $4.8441 \times 10^{-1}$ \\
$\mathrm{NO}$ & $2.2660 \times 10^{-2}$ & $1.0900 \times 10^{-3}$ \\
\hline
\end{tabular}




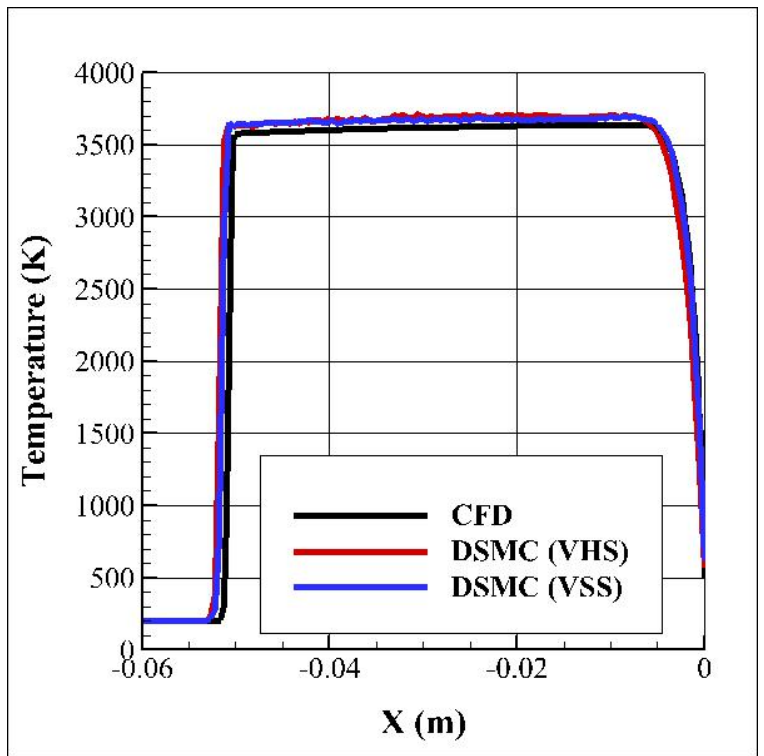

(a) Mach 10 oxygen

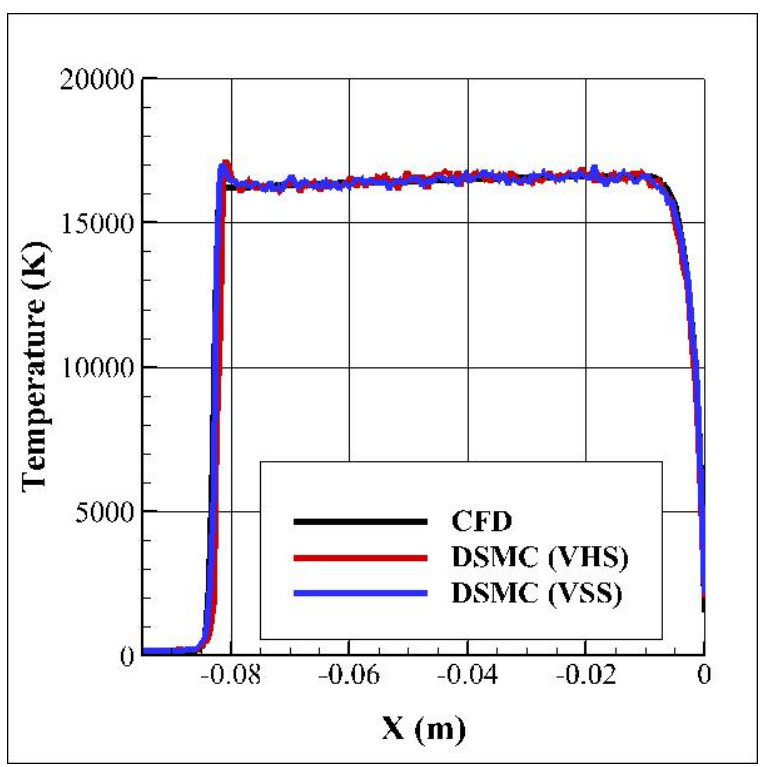

(b) Mach 25 oxygen

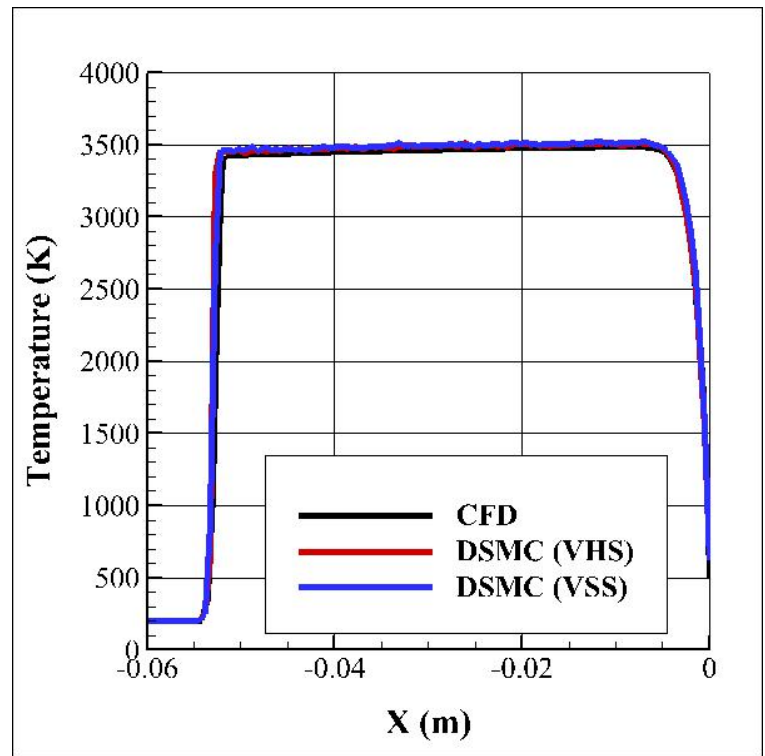

(c) Mach 10 air

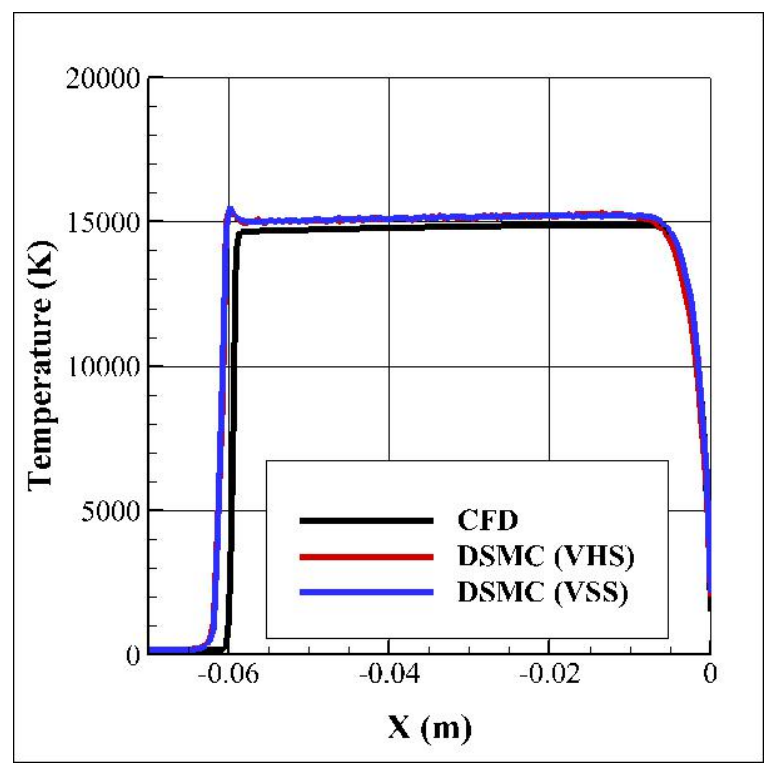

(d) Mach 25 air

FIGURE 1. Stagnation streamline distribution of total temperature.

\section{Flow Field Structure and Temperature}

The distributions of total temperature along the stagnation stream line are shown in Figure 1 for all of the conditions considered. The DSMC shock standoff distances and temperature profiles compare well with the CFD predictions for both the VHS and VSS model parameters. 


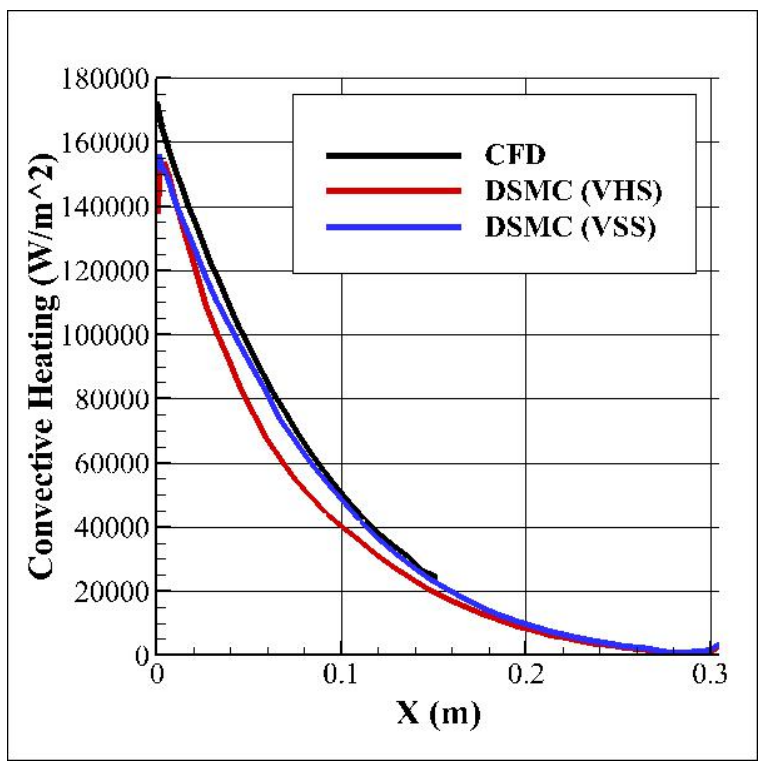

(a) Mach 10 oxygen

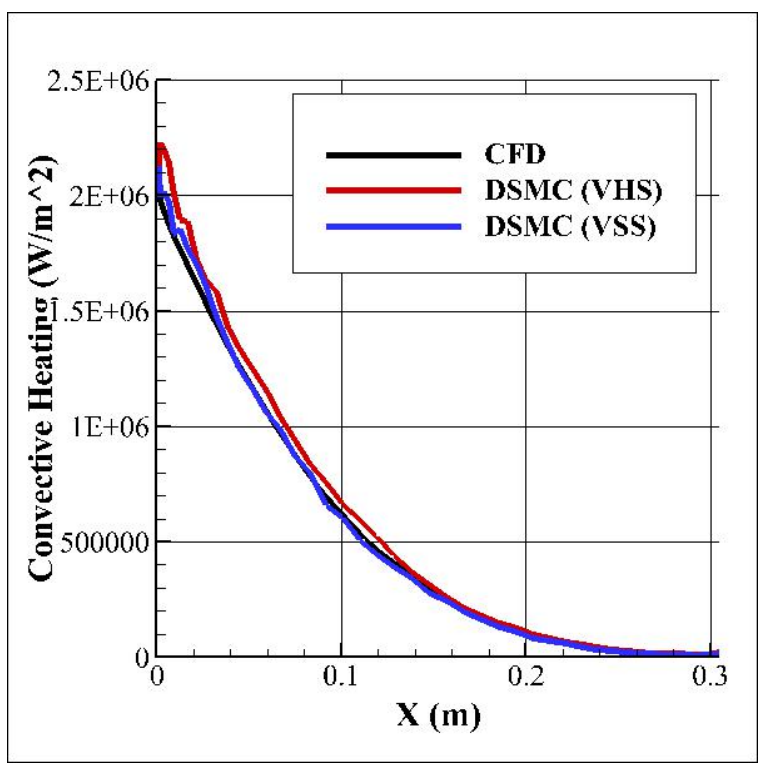

(b) Mach 25 oxygen

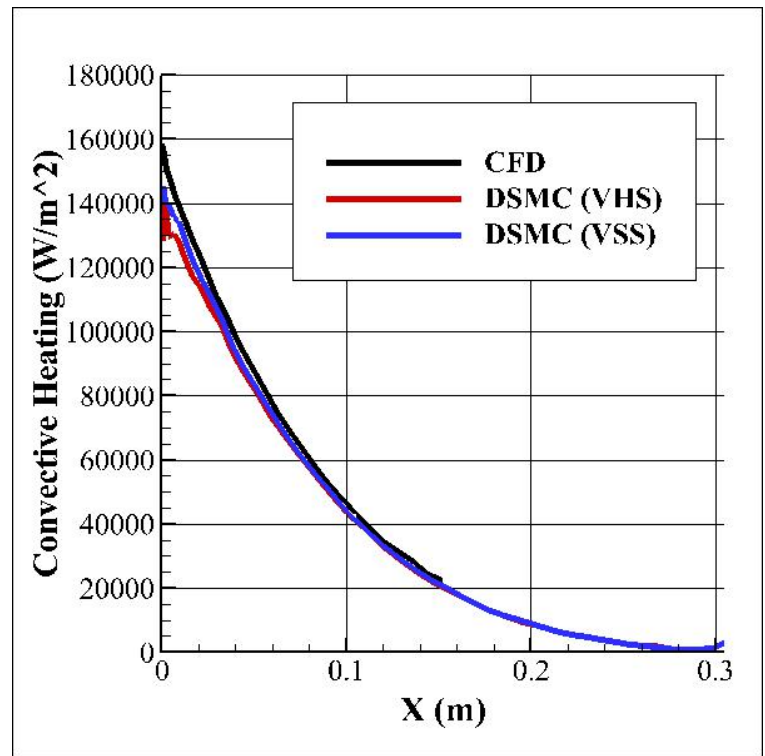

(c) Mach 10 air

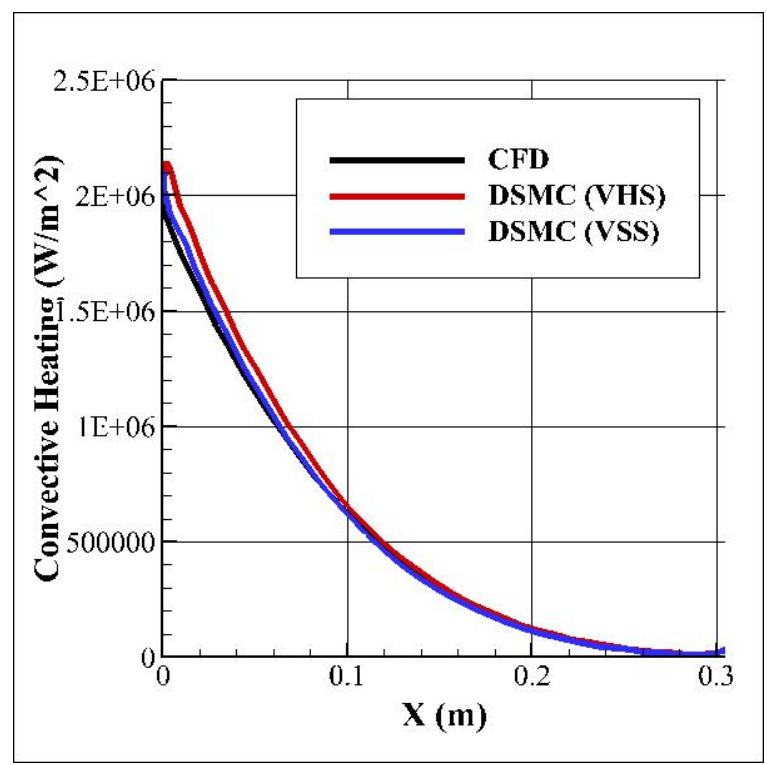

(d) Mach 25 air

FIGURE 2. Surface heating distributions.

\section{Surface Heating}

The surface heating distributions over the surface of the cylinder are presented in Figure 2. For all conditions, the VSS model parameter values more closely match those predicted by the CFD solutions. The VHS results underpredict the heating for the Mach 10 cases and then over-predict the heating for the Mach 25 cases. 


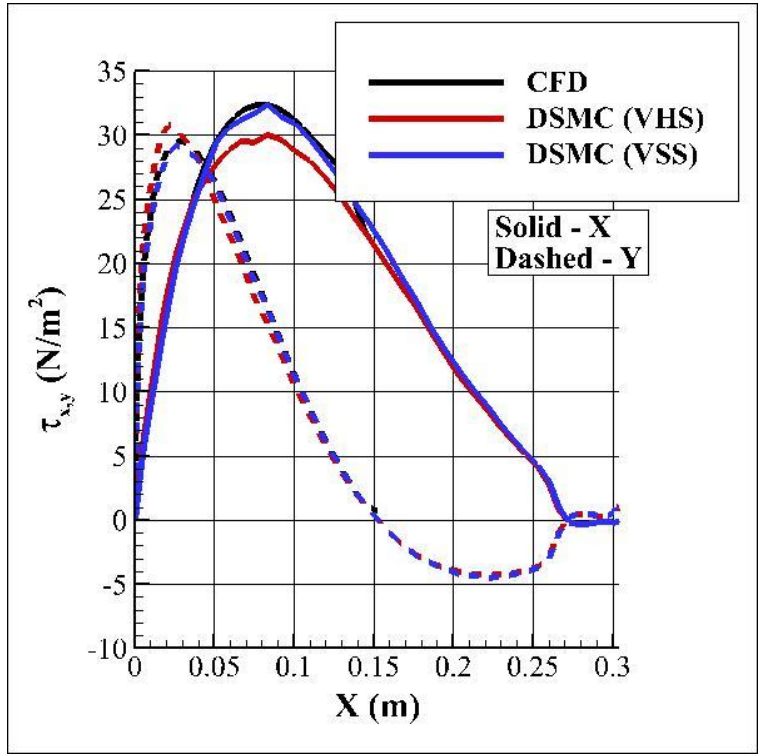

(a) Mach 10 oxygen

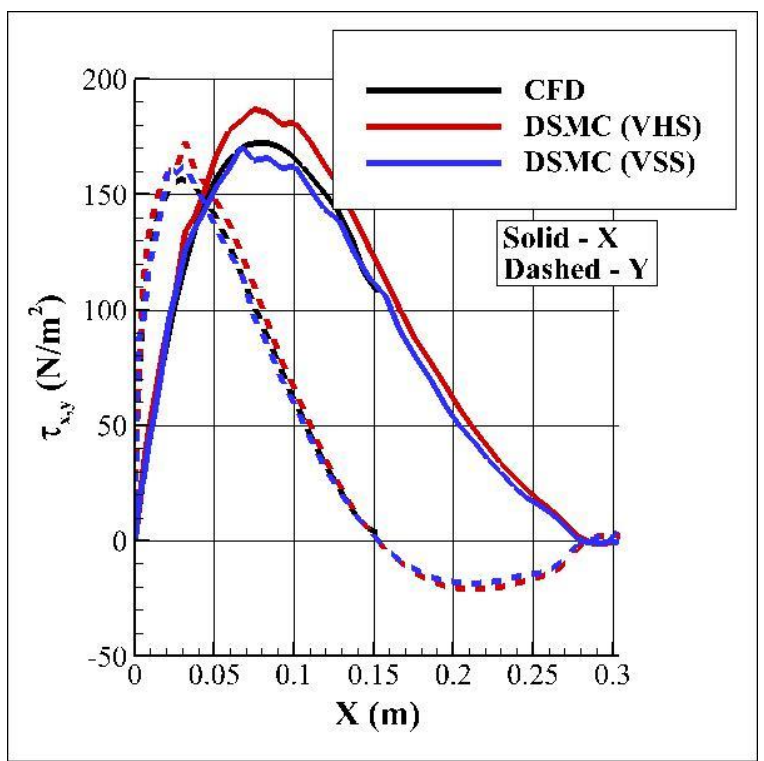

(b) Mach 25 oxygen

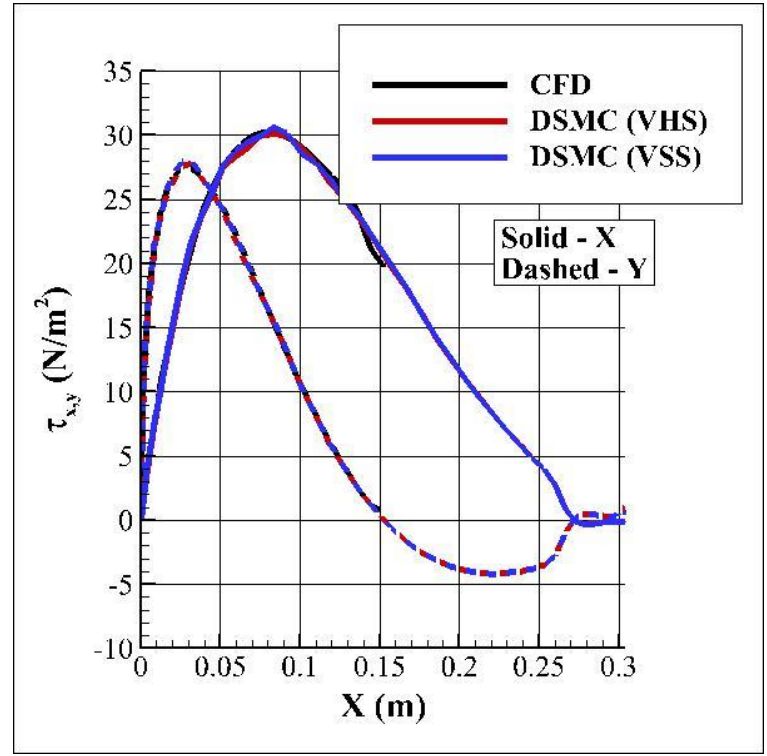

(c) Mach 10 air

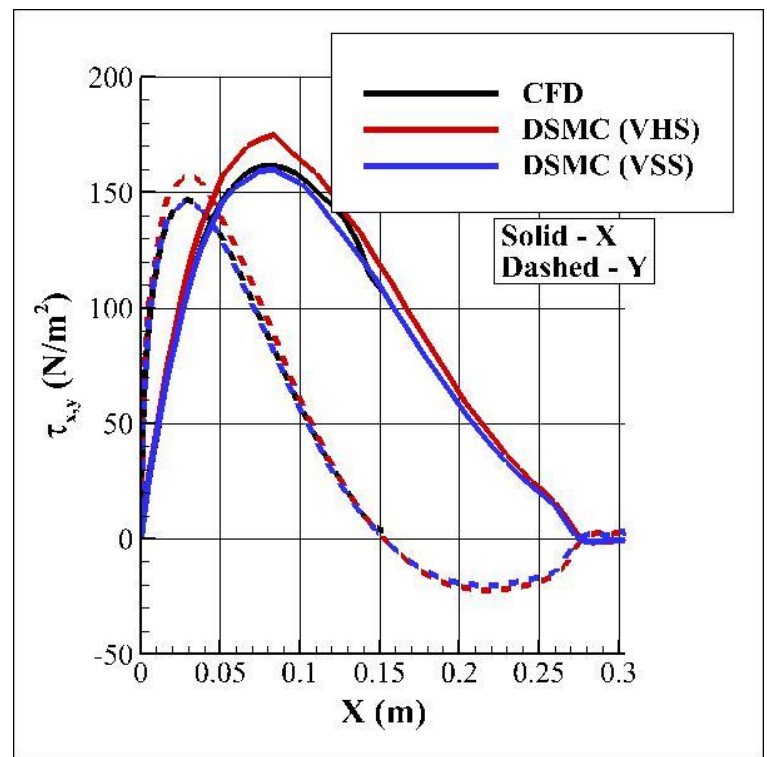

(d) Mach 25 air

FIGURE 3. Surface shear stress distributions.

\section{Surface Shear Stress}

The surface shear stress distributions over the surface of the cylinder are presented in Figure 3. For all conditions, the VSS model parameter values more closely match those predicted by the CFD solutions. 


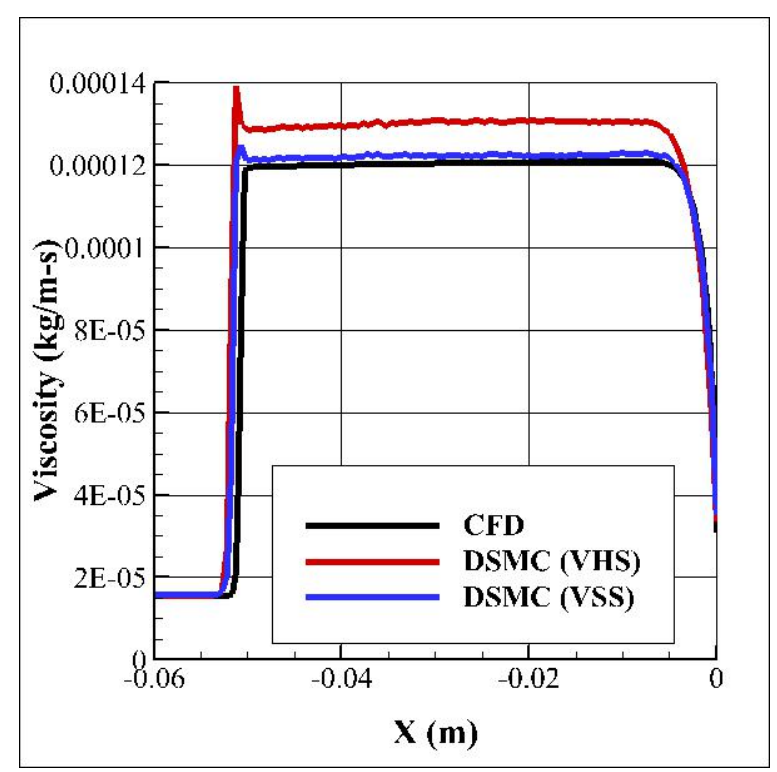

(a) Mach 10 oxygen

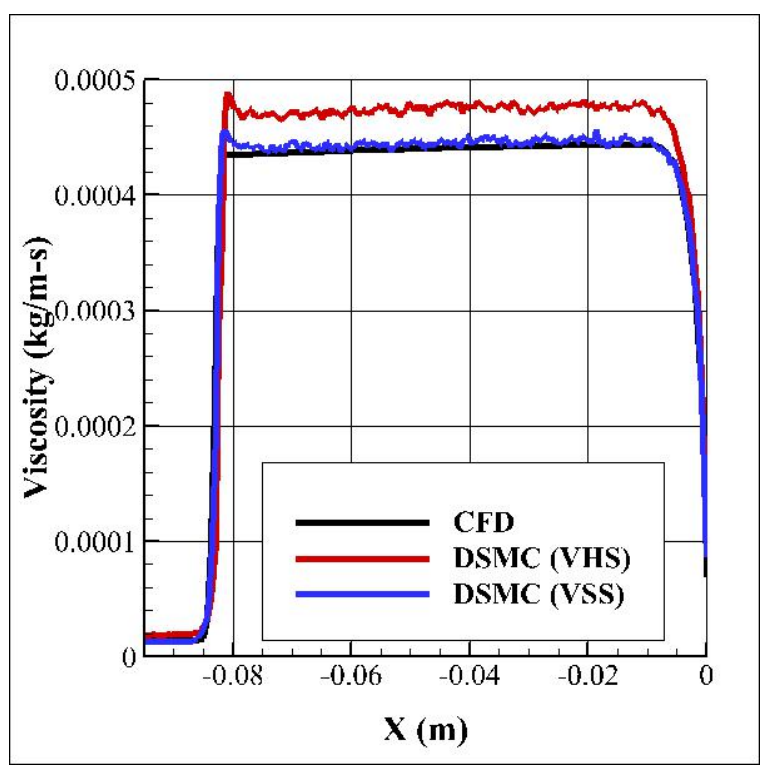

(b) Mach 25 oxygen

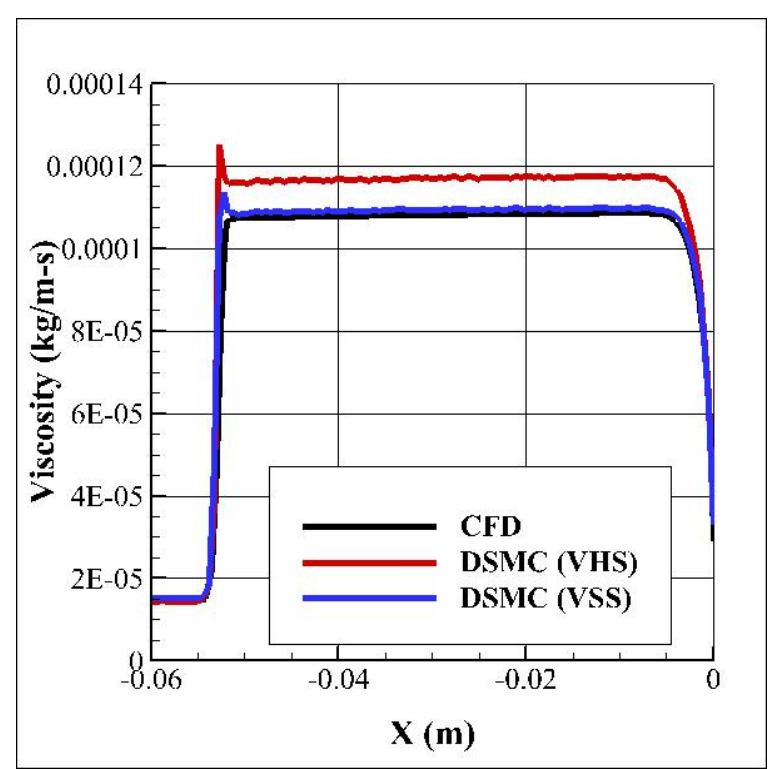

(c) Mach 10 air

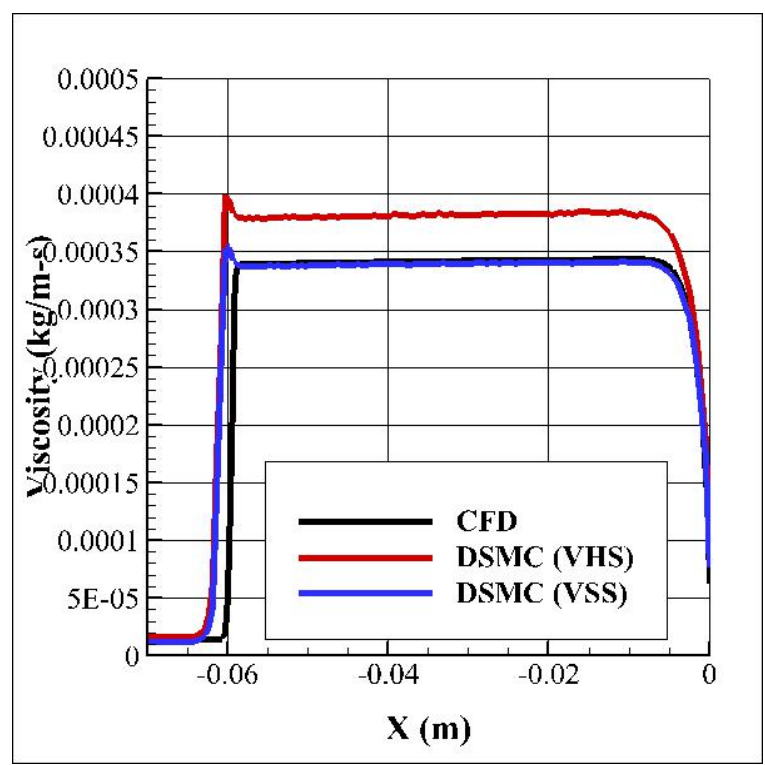

(d) Mach 25 air

FIGURE 4. Stagnation streamline distribution of mixture viscosity.

\section{Mixture Viscosity}

The distributions of mixture viscosity along the stagnation stream line are shown in Figure 4 for all of the conditions considered. When the values of mixture viscosity are compared to the CFD prediction, the VSS model parameter values reproduce the CFD predictions, as expected, while the VHS model parameter values over-predict the mixture viscosity. 


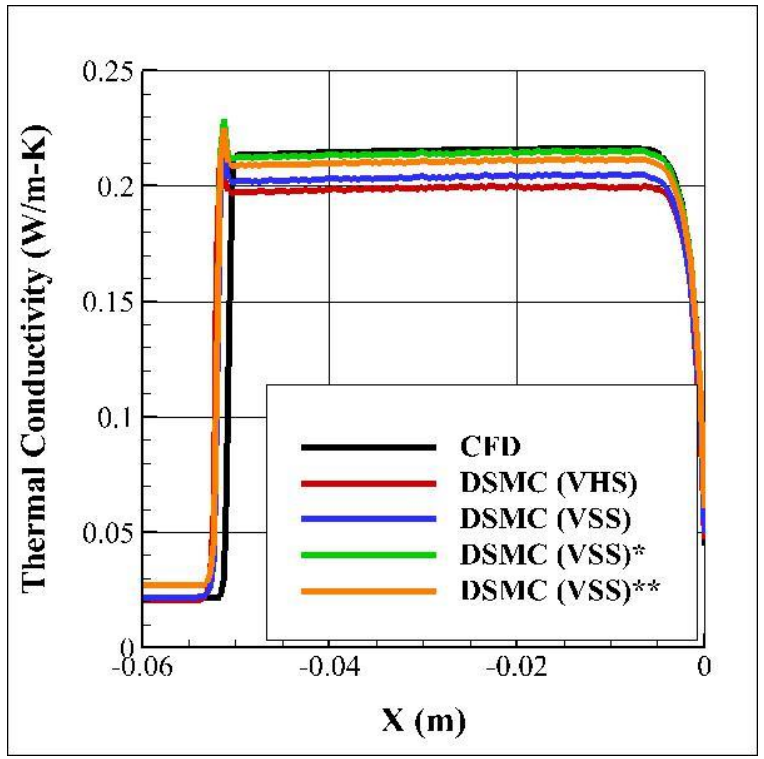

(a) Mach 10 oxygen

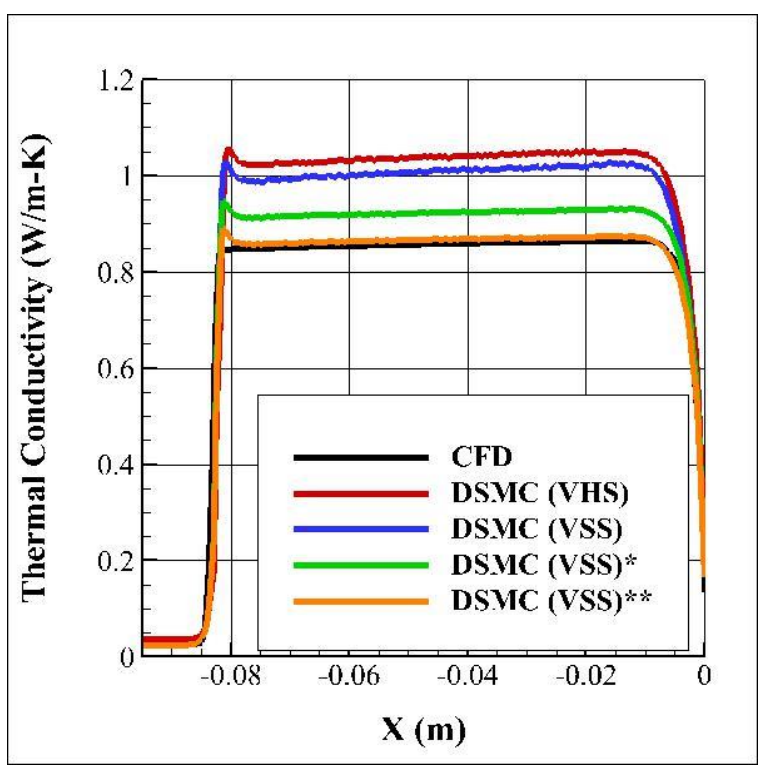

(b) Mach 25 oxygen

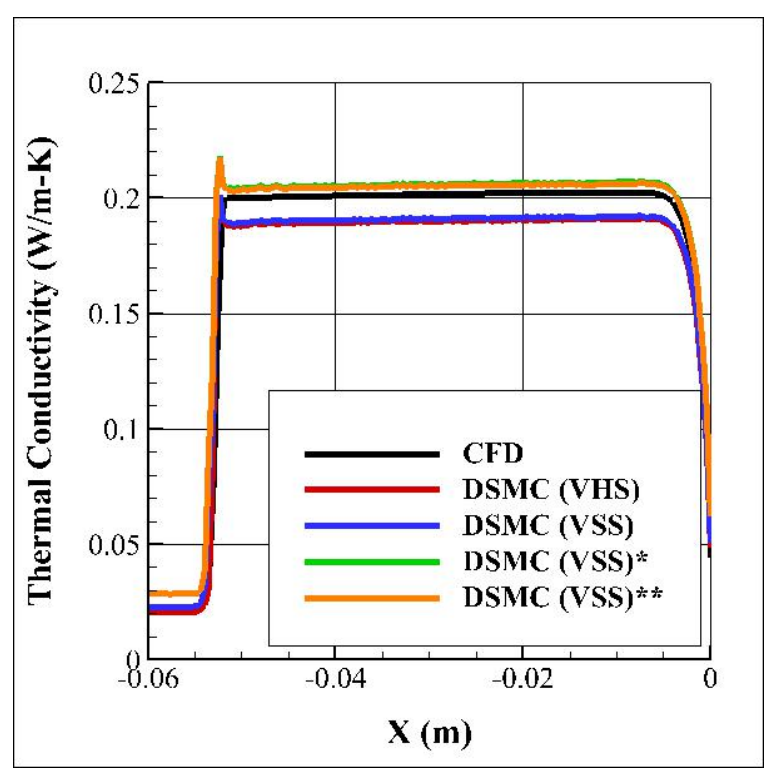

(c) Mach 10 air

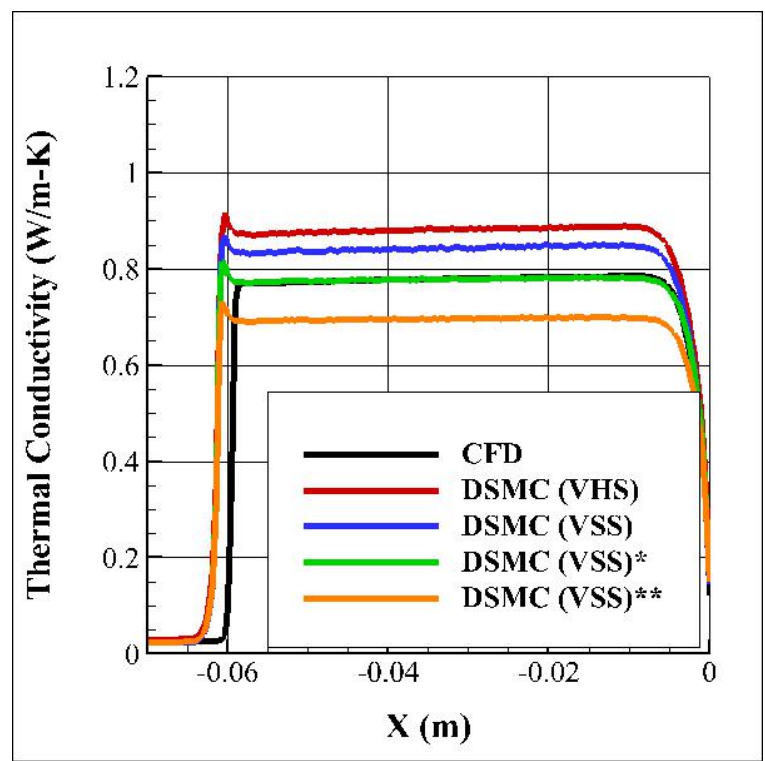

(d) Mach 25 air

FIGURE 5. Stagnation streamline distribution of mixture thermal conductivity.

\section{Mixture Thermal Conductivity}

The distributions of mixture thermal conductivity along the stagnation stream line are shown in Figure 5 for all of the conditions considered. When thermal conductivity is examined, neither the VHS nor the VSS models accurately reproduced the expected values represented by the CFD predictions for any of the conditions considered using the full description of mixture thermal conductivity defined above. This is because of the assumptions used in calculating the CFD mixture thermal conductivity. As described in Ref. 20, it is assumed that the vibrational component of thermal conductivity is equal to the rotational component in LAURA. Further, it is not discussed how the electronic 
component is calculated. Therefore, two extra lines are added to the results in Figure 5. The DSMC (VSS)* and DSMC (VSS)** lines are defined as

$$
\begin{gathered}
K_{\text {total }}^{*}=K_{t r}+2 K_{\text {rot }}+K_{e l}^{\prime} \\
K_{\text {total }}^{* *}=K_{t r}+2 K_{\text {rot }}
\end{gathered}
$$

where $K_{e l}^{\prime}$ is the electronic thermal conductivity using the sum over only the first two energy levels from Eq. (26). The only difference between Eq. (27) and (28) is the addition of the electronic energy component in Eq. (27). If we examine the Mach 10 oxygen and air conditions, it is clear that the addition of the electronic energy component makes little difference in the results because the temperature is not high enough to have a significant population in the first excited energy level. For these cases, the modified thermal conductivity described by Eq. (27) matches the predicted CFD values reasonably well.

When considering the Mach 25 oxygen and air conditions, the modified values of thermal conductivity do match the predicted CFD values, but Eq. (28) matches for the oxygen condition and Eq. (27) matches for the air condition. This is because, for the oxygen case, the flow is entirely atomic oxygen and although atomic oxygen has electronic energy, the CFD code does not consider it because of the assumption of the two-temperature model. Therefore, we are able to reproduce the mixture thermal conductivities for all four conditions when taking into account the assumptions that are made by the CFD code.

\section{CONCLUSION}

Previously calibrated VSS collision model parameters have been successfully implemented in the MAP DSMC code and have been compared to one of the CFD codes (LAURA) from which the collision integrals were used to perform the calibrations. In general, the resultant shock standoff distance, total temperature distribution, and mixture viscosity along the stagnation streamline of the 2D cylinder compared very favorably for both Mach numbers and gas compositions presented herein. However, discrepancies were found when the mixture thermal conductivity was examined. In order to resolve these discrepancies, the assumptions that were made in the CFD code were applied to the post-processing of the DSMC results. When the assumptions of summing over only the first two electronic energy levels and not including the electronic energy component for atomic species were applied, the DSMC VSS results were able to reproduce the predicted CFD values.

The results of the calibrated VSS parameters were also compared to previously recommended VHS collision model parameters. It was found that, while both models were equally good at predicting the shock standoff distance and the total temperature distribution, the VHS model parameters could not predict the mixture viscosity correctly and did not predict the surface quantities of interest as well as the VSS model parameters.

\section{REFERENCES}

1. T.E. Schwartzentruber, I.D. Boyd, Journal of Computational Physics, 225, 1159-1174 (2007)

2. J.-S. Wu, Y.-Y. Lian, G. Cheng, R.P. Koomullil, K.-C. Tseng, Journal of Computational Physics, 219, 579-607 (2006)

3. J.L. Papp, R.G. Wilmoth, C.C. Chartrand, S.M. Dash, AIAA Paper 2006-4112

4. K.A. Stephani, D.B. Goldstein, P.L. Varghese, Journal of Computational Physics, 232, 468-481 (2013)

5. K. Swaminathan-Goplan, K.A. Stephani, Physics of Fluids, 28, 027101 (2016)

6. K. Swaminathan-Goplan, S. Subramaniam, K.A. Stephani, Phys. Rev. Fluids, 1, 083402 (2017)

7. A.J. Lofthouse, L.C. Scalabrin, I.D. Boyd, AIAA Paper 2007-3903

8. D.S. Liechty, Journal of Spacecraft and Rockets, 52(6), 1521-1529 (2015)

9. A. Mazaheri, P.A. Gnoffo, C.O. Johnston, B. Kleb, NASA TM 2010-216836

10. M.J. Wright, D. Bose, G.E. Palmer, E. Levin, AIAA Journal, 43(12), 2558-2564 (2005)

11. G.E. Palmer, M.J. Wright, Journal of Thermophysics and Heat Transfer, 17(2), 232-239 (2003)

12. G. Bird, Molecular Gas Dynamics and the Direct Simulation of Gas Flows (Oxford University Press, Oxford, 1994).

13. K. Koura, H. Matsumoto, Physics of Fluids A 4 (5), 1083-1085 (1992).

14. K.A. Stephani, D.B. Goldstein, P.L. Varghese, Physics of Fluids, 24, 077101 (2012)

15. S. Gordon, B.J. McBride, NASA RP 1311 
16. R.G. Wilmoth, G.J. LeBeau, A.B. Carlson, AIAA Paper 1996-1812

17. G.J. LeBeau, F.E. Lumpkin III, Computer Methods in Applied Mechanics and Engineering 191 (6-7), 595-609 (2001)

18. G.A. Bird, Molecular Gas Dynamics and the Direct Simulation of Gas Flows (Oxford University Press, New York, 1994)

19. W.G. Vincenti, C.H. Kruger, Jr., Introduction to Physical Gas Dynamics (Krieger Publishing Company, Malabar, 1965)

20. P.A. Gnoffo, R.N. Gupta, J.L. Shinn, NASA TP 2867 